\title{
Relaxed Matching for Stabilization of Mechanical Systems
}

\author{
D.A. Long*, A.M.Bloch ${ }^{\dagger}$, J.E. Marsden ${ }^{\ddagger}$, \\ and D.V. Zenkov*
}

Keywords: Controlled Lagrangians, kinetic shaping

\begin{abstract}
The method of controlled Lagrangians is a technique for stabilizing relative equilibria of mechanical systems with symmetry. This paper develops a modification of kinetic shaping technique in the theory of controlled Lagrangians. The main idea is that the dynamics associated with the controlled Lagrangian is amended by non-conservative forces that act in the shape directions; this gives additional freedom and hence applicability to the method. The usefulness of this methodology is demonstrated on the pendulum on a rotor arm.
\end{abstract}

\section{Introduction}

The method of controlled Lagrangians for stabilization of relative equilibria (steady state motions) originated in Bloch, Leonard, and Marsden [6] and was then developed in Auckly [2], Bloch, Leonard, and Marsden [7, 9, 10], Bloch, Chang, Leonard, and Marsden [11], and Hamberg [14, 15]. A similar approach for Hamiltonian controlled systems was introduced and further studied in the work of Blankenstein, Ortega, van der Schaft, Maschke, Spong, and their collaborators (see, e.g., [19, 20], and related references). The two methods were shown to be equivalent in [12]. For related results on applications of generalized canonical transformations to stabilization of Hamiltonian systems see [13] and references therein. A nonholonomic version of the method of controlled Lagrangians was developed in [22, 23], and [3].

\footnotetext{
*Department of Mathematics, North Carolina State University, Raleigh, NC 27695

${ }^{\dagger}$ Department of Mathematics, University of Michigan, Ann Arbor, MI 48109

${ }^{\ddagger}$ Control and Dynamical Systems, California Institute of Technology, Pasadena, CA 91125
} 
The method was extended to the discrete setting in [4] and [5].

In the controlled Lagrangian approach, one considers a mechanical system with an uncontrolled (free) Lagrangian equal to kinetic energy minus potential energy. This Lagrangian is assumed to be invariant with respect to the action of a Lie group $G$ on the configuration space. In order to stabilize a relative equilibrium, one technique is to modify the kinetic energy to produce a controlled Lagrangian which describes the dynamics of the controlled closed-loop system. The equations corresponding to this controlled Lagrangian are the closed-loop equations. The new terms appearing in those equations corresponding to the directly controlled variables are interpreted as control inputs. The modifications to the Lagrangian are chosen so that no new terms appear in the equations corresponding to the variables that are not directly controlled. We refer to this process as kinetic shaping. Once the form of the control law is derived using the controlled Lagrangian, the stability of a relative equilibrium of the closed-loop system can be determined by energy methods, using any available freedom in the choice of the parameters of the controlled Lagrangian. To obtain asymptotic stabilization, dissipation-emulating terms are added to the control input.

The method is extended in [11] to the class of Lagrangian mechanical systems with potential energy that may break symmetry, i.e., there is still a symmetry group $G$ for the kinetic energy of the system but one may now have a potential energy that need not be $G$-invariant.

In order to proceed with kinetic shaping, one needs to verify the matching conditions that ensure that the original controlled mechanical system is identical to the system associated with the controlled Lagrangian. These conditions restrict the choice of the modified kinetic energy. It is not always possible to satisfy the matching conditions (see $[2,7,9,10,11]$ for details). Thus, it is not always possible to construct a stabilizing controller using the method of controlled Lagrangians for an underactuated system.

In this paper we develop the following modification of kinetic shaping: The dynamics associated with the controlled Lagrangian is amended by non-conservative forces that act in the shape directions. As in the method of controlled Lagrangian, we require that this dynamics is identical to the original controlled dynamics. We show that our approach is less restrictive than the original matching techniques since we have more freedom in modifying the kinetic energy. This flexibility targets two goals. One is to be able to perform kinetic shaping on systems with incompatible matching conditions. The other is to enlarge the set of stabilizing controllers that can be constructed by the method of controlled Lagrangians. The latter is illustrated with the controller with a larger stabilization region than the controller obtained by the original matching algorithm.

In a forthcoming publication we intend to extend our formalism to systems with nonabelian symmetries and to full state space stabilization problems.

The paper is organized as follows: In Section 2 we review the method of controlled Lagrangians for stabilization of relative equilibria of mechanical systems. The main results of the paper are exposed in Section 3. The theoretical analysis is validated by simulating the inverted pendulum on a rotor arm in Section 4 . 


\section{Matching and Controlled Lagrangians}

In this section we briefly review some of the results of Bloch, Leonard, and Marsden $[10]$.

Assume that an abelian Lie group $G$ acts freely and properly on a configuration manifold $Q$. Let $S$ denote the shape space $Q / G$. The configuration variables are written as $(\phi, s)$, where $\phi=\left(\phi^{1}, \ldots, \phi^{m}\right)$ are local coordinates on $S$ and $s=\left(s^{1}, \ldots, s^{n}\right) \in G$. The velocity phase space, $T Q$, has coordinates $(\phi, s, \dot{\phi}, \dot{s})=\left(\phi^{\alpha}, s^{a}, \dot{\phi}^{\alpha}, \dot{s}^{a}\right)$. We assume that the Lagrangian is $G$-invariant and reads

$$
L(\phi, \dot{\phi}, \dot{s})=\frac{1}{2}\left(g_{\alpha \beta}(\phi) \dot{\phi}^{\alpha} \dot{\phi}^{\beta}+2 g_{\alpha a}(\phi) \dot{\phi}^{\alpha} \dot{s}^{a}+g_{a b}(\phi) \dot{s}^{a} \dot{s}^{b}\right)-U(\phi)
$$

and the corresponding controlled dynamics is

$$
\begin{aligned}
\frac{d}{d t} \frac{\partial L}{\partial \dot{\phi}^{\alpha}}-\frac{\partial L}{\partial \phi^{\alpha}} & =0 \\
\frac{d}{d t} \frac{\partial L}{\partial \dot{s}^{a}} & =u_{a}
\end{aligned}
$$

where $u_{a}$ are the components of the control input $u$.

Recall that a relative equilibrium of a system with abelian symmetry is a solution with zero shape and constant group velocity components. We assume that the relative equilibria $\phi=\phi_{e}, \dot{s}=$ const of uncontrolled system (1) and (2) are unstable. In the rest of the paper we assume that $\phi_{e}=0$, which can always be accomplished by an appropriate choice of local coordinates for each relative equilibrium.

In order to stabilize the relative equilibria $\phi=0, \dot{s}=$ const, Bloch, Leonard, and Marsden [10] define the controlled Lagrangian by

$$
L_{\tau, \sigma}(\phi, \dot{\phi}, \dot{s})=L\left(\phi^{\alpha}, \dot{\phi}^{\beta}, \dot{s}^{a}+\tau_{\alpha}^{a}(\phi) \dot{\phi}^{\alpha}\right)+\frac{1}{2} \sigma_{a b}(\phi) \tau_{\alpha}^{a}(\phi) \tau_{\beta}^{b}(\phi) \dot{\phi}^{\alpha} \dot{\phi}^{\beta}
$$

and require that the Euler-Lagrange equations for $L_{\tau, \sigma}$ are identical to equations (1) and (2).

Theorem 1 ([10]). The controlled Euler-Lagrange equations (1) and (2) coincide with the Euler-Lagrange equations for the controlled Lagrangian (3) if

$$
u_{a}=-\frac{d}{d t}\left(g_{a b} \tau_{\alpha}^{b} \dot{\phi}^{\alpha}\right)
$$

and the following matching conditions hold:

$$
\sigma_{a b} \tau_{\alpha}^{b}=-g_{\alpha a}, \quad \sigma^{b d}\left(\sigma_{a d, \alpha}+g_{a d, \alpha}\right)=2 g^{b d} g_{a d, \alpha}, \quad \tau_{\alpha, \delta}^{b}-\tau_{\delta, \alpha}^{b}-g^{d b} g_{a d, \alpha} \tau_{\delta}^{a}=0
$$

The velocity shift $\dot{s}^{a} \rightarrow \dot{s}^{a}+\tau_{\alpha}^{a}(\phi) \dot{\phi}^{\alpha}$ corresponds to a new choice of the horizontal space in $T Q$, while the last term in (3) changes the metric along the vertical (i.e., tangent to the group orbit) direction (see [10] for details). The quantities 
$\tau_{\alpha}^{a}(\phi)$ and $\sigma_{a b}(\phi)$ are selected in such a way that the relative equilibria of interest become orbitally stable, that is, one observes stability relative to the variables $\phi^{\alpha}$, $\dot{\phi}^{\alpha}$, and $\dot{s}^{a}$, but not relative to $s^{a}$. See [10] for details.

Matching conditions (4) restrict the set of available control inputs and may even be incompatible. In this paper we introduce an alternative approach to the problem of stabilization of relative equilibria of (1) and (2). This approach can be carried out when the matching conditions (4) cannot be resolved.

\section{Relaxed Matching}

\subsection{Matching with Shape Forcing}

The key idea of relaxing matching techniques, proposed in [16], is to introduce a non-conservative force in the shape equation associated with the controlled Lagrangian (3). That is, the dynamics associated with (3) is

$$
\begin{aligned}
\frac{d}{d t} \frac{\partial L_{\tau, \sigma}}{\partial \dot{\phi}^{\alpha}}-\frac{\partial L_{\tau, \sigma}}{\partial \phi^{\alpha}} & =w_{\alpha}, \\
\frac{d}{d t} \frac{\partial L_{\tau, \sigma}}{\partial \dot{s}^{a}} & =u_{a}^{\mathrm{diss}}
\end{aligned}
$$

where $u_{a}^{\text {diss }}$ is the dissipation-emulating term that is necessary for asymptotic stabilization. In the rest of the paper, $g^{\alpha \beta}$ and $g^{a b}$ denote the inverses of the matrices $g_{\alpha \beta}$ and $g_{a b}$.

Equation (6) is equivalent to

$$
\frac{d}{d t} \tilde{J}_{a}=u_{a}^{\text {diss }}
$$

where

$$
\tilde{J}_{a}=\frac{\partial L_{\tau, \sigma}}{\partial \dot{s}^{a}}=g_{\alpha a} \dot{\phi}^{\alpha}+g_{a b} \dot{s}^{b}+g_{a b} \tau_{\alpha}^{b} \dot{\phi}^{\alpha}
$$

is the controlled momentum.

Theorem 2. The controlled Euler-Lagrange equations (1) and (2) coincide with equations (5) and (6) associated with the controlled Lagrangian (3) if

$$
\begin{aligned}
& u_{a}=u_{a}^{\text {cons }}+u_{a}^{\text {diss }}, \\
& u_{a}^{\mathrm{cons}}=-\frac{d}{d t}\left(g_{a b} \tau_{\alpha}^{b} \dot{\phi}^{\alpha}\right) \text {, } \\
& w_{\alpha}=\left(g_{\alpha a} \tau_{\beta}^{a}+\sigma_{a b} \tau_{\alpha}^{a} \tau_{\beta}^{b}\right) \ddot{\phi}^{\beta}+\tau_{\alpha}^{a} u_{a}^{\text {diss }}+\left(\partial_{\gamma}\left(\tau_{\alpha}^{a}\right) \tilde{J}_{a}-g^{b c} \tilde{J}_{c} \partial_{\alpha}\left(g_{a b} \tau_{\gamma}^{a}\right)\right) \dot{\phi}^{\gamma} \\
& +\left[\partial_{\gamma}\left(g_{\alpha a} \tau_{\beta}^{a}+\sigma_{a b} \tau_{\alpha}^{a} \tau_{\beta}^{b}\right)-\partial_{\alpha}\left(g_{\gamma a} \tau_{\beta}^{a}+\frac{1}{2} g_{a b} \tau_{\gamma}^{a} \tau_{\beta}^{b}+\frac{1}{2} \sigma_{a b} \tau_{\gamma}^{a} \tau_{\beta}^{b}\right)\right. \\
& \left.+\partial_{\alpha}\left(g_{a b} \tau_{\beta}^{a}\right) g^{b c} g_{\gamma c}+\partial_{\alpha}\left(g_{a b} \tau_{\beta}^{a}\right) \tau_{\gamma}^{b}\right] \dot{\phi}^{\beta} \dot{\phi}^{\gamma} \text {. }
\end{aligned}
$$

Proof. We start by asking that equation (2) be identical to equation (6). This is 
accomplished by setting

$$
u_{a}^{\text {cons }}=\frac{d}{d t} \frac{\partial L}{\partial \dot{s}^{a}}-\frac{d}{d t} \frac{\partial L_{\tau, \sigma}}{\partial \dot{s}^{a}} .
$$

Straightforward calculation shows that this requirement is equivalent to condition (8).

To finish the proof, we require that equations (1) and (5) are the same. This defines the term $w_{\alpha}$ by the formula

$$
w_{\alpha}=\left(\frac{d}{d t} \frac{\partial L_{\tau, \sigma}}{\partial \dot{\phi}^{\alpha}}-\frac{\partial L_{\tau, \sigma}}{\partial \phi^{\alpha}}\right)-\left(\frac{d}{d t} \frac{\partial L}{\partial \dot{\phi}^{\alpha}}-\frac{\partial L}{\partial \phi^{\alpha}}\right) .
$$

Formula (10) represents $w_{\alpha}$ as a function of $(r, \dot{r}, \dot{s})$. Using the Legendre transform on $T G$, we obtain formula $(9)$ that represents $w_{\alpha}$ as a function of $\left(r, \dot{r}, \widetilde{J}_{a}\right)$.

Remark. Condition (9) replaces the matching conditions (4).

\subsection{Asymptotic Stabilization of Relative Equilibria}

In this section we suggest two Lyapunov function design strategies. In the rest of the paper we restrict the exposition to the instance of one shape and one group degree of freedom. The general setting will be discussed elsewhere. We further assume that the configuration space $Q$ is the direct product of a one-dimensional shape space $S$ and a one-dimensional Lie group $G$ and the Lagrangian is represented by the formula ${ }^{1}$

$$
L(\phi, \dot{\phi}, \dot{s})=\frac{1}{2}\left(\alpha \dot{\phi}^{2}+2 \beta(\phi) \dot{\phi} \dot{s}+\gamma(\phi) \dot{s}^{2}\right)-U(\phi)
$$

The first method, which is only outlined here, is inspired by the approach developed in [10]. The energy restricted to the controlled momentum level $\widetilde{J}=\mu$ through the relative equilibrium of interest is given by the formula

$$
E_{\mu}=\frac{1}{2}\left(\alpha-\frac{\beta^{2}(\phi)}{\gamma(\phi)}-\beta(\phi) \tau(\phi)\right) \dot{\phi}^{2}+\frac{\mu^{2}}{2 \gamma(\phi)}+U(\phi) .
$$

Assuming that $\gamma=$ const,

$$
\dot{E}_{\mu}=-\frac{1}{2}\left(\beta^{\prime}(\phi) \tau(\phi)-\beta(\phi) \tau^{\prime}(\phi)\right) \dot{\phi}^{3}-\frac{\beta(\phi)}{\gamma} u^{\mathrm{diss}} \dot{\phi}
$$

Using the result of Oziraner [21], we conclude that the relative equilibrium is asymptotically stable with respect to $(\phi, \dot{\phi})$ when $U(\phi)$ is negative definite, $\alpha-\frac{\beta^{2}}{\gamma}-\beta \tau<0$, and $u^{\text {diss }}=c(\phi) \dot{\phi}$ where $c(\phi)<0$.

\footnotetext{
${ }^{1}$ Here, the coefficient $\alpha$ in (11) is assumed to be independent of $\phi$. This is done to simplify the exposition.
} 
Method 1 can be shown to work when $\alpha$ is not a constant but fails to work for non-constant $\gamma$.

For the second method we merely assume from the beginning that $u^{\text {diss }}=$ $c(\phi) \dot{\phi}$. Let $C(\phi)$ be the antiderivative of $c(\phi)$ that vanishes at $\phi=\phi_{e}$. The second method does not require that $\gamma=$ const. However, the existence of an antiderivative of $c(\phi)$ may be problematic in the multidimensional case. Multidimensional generalizations for both methods will be studied in a forthcoming publication.

The control law of Theorem 2 takes the form:

$$
u=-\frac{d}{d t}(\gamma(\phi) \tau(\phi) \dot{\phi})+c(\phi) \dot{\phi}
$$

In addition, equation (7) results in a conservation law:

$$
\widetilde{J}-C(\phi)=p,
$$

where $\widetilde{J}$ is the controlled momentum, and the constant $p$ labels the levels of the conservation law (12).

Define the reduced Lagrangian by the formula

$$
l_{p}(\phi, \dot{\phi})=\frac{1}{2}\left(\alpha-\frac{\beta^{2}(\phi)}{\gamma(\phi)}-\beta(\phi) \tau(\phi)\right) \dot{\phi}^{2}-U_{p}(\phi)
$$

where

$$
U_{p}(\phi)=U(\phi)-\int_{0}^{\phi} \frac{\gamma^{\prime}(x)(C(x)+p)^{2}}{2 \gamma^{2}(x)} d x
$$

is the amended potential. Let $f$ be a (non-conservative) force defined by

$$
f=-\frac{\gamma^{\prime}}{\gamma}(p+C) \tau \dot{\phi}+\left(\frac{\beta \tau^{\prime}-\beta^{\prime} \tau}{2}+\frac{\gamma^{\prime} \tau^{2}}{2}+\frac{\beta \gamma^{\prime} \tau}{\gamma}\right) \dot{\phi}^{2}
$$

The following statement is obtained by a straightforward caclulation.

Theorem 3. The dynamics defined by (5) and (6) reduced to level sets of conservation law (12) is given by the forced Euler-Lagrange equations for the reduced Lagrangian (13),

$$
\frac{d}{d t} \frac{\partial l_{p}}{\partial \dot{\phi}}-\frac{\partial l_{p}}{\partial \phi}=-\frac{\beta c}{\gamma} \dot{\phi}+f
$$

Remark. Neither the reduced Lagrangian, nor control input (8) depends on the term $\frac{1}{2} \sigma(\tau \dot{\phi})^{2}$ in the controlled Lagrangian (3). Thus, without loss of generality we can set $\sigma=0$. Of course, in the case when the standard matching techniques are applicable, one may be motivated to select $\sigma$ that satisfies the matching conditions (4) as this value of $\sigma$ eliminates the force $f$ in (14).

Recall that $\phi=0, \dot{s}=$ const are relative equilibria of (1) and (2). That is, $\phi=0$ is an equilibrium of the reduced shape equation (14). If this equilibrium is stable, the corresponding relative equilibria of (1) and (2) are orbitally stable. 
The energy associated with Lagrangian (13) is

$$
E_{p}(\phi, \dot{\phi})=\frac{1}{2}\left(\alpha-\frac{\beta^{2}}{\gamma}-\beta \tau\right) \dot{\phi}^{2}+U_{p} .
$$

We now show that (15) can be used as a Lyapunov function for stability analysis of relative equilibria $\phi=0, \dot{s}=$ const.

The relative equilibria of interest of the uncontrolled system are unstable, which implies $U^{\prime \prime}(0)-p^{2} \gamma^{\prime \prime}(0) / 2 \gamma^{2}(0) \leq 0$. We assume here that $U_{p}^{\prime \prime}(0)$ is negative. ${ }^{2}$ We then select $\tau(\phi)$ such that

$$
\alpha-\frac{\beta^{2}}{\gamma}-\beta \tau<0,
$$

which makes reduced energy (15) negative-definite in a neighborhood of the equilibrium of interest. The flow derivative of $E_{p}$ is

$$
\dot{E}_{p}=-\frac{\beta c}{\gamma} \dot{\phi}^{2}+f \dot{\phi}
$$

Thus, selecting $c(\phi)$ such that

$$
\frac{\beta c}{\gamma}+\frac{\gamma^{\prime}(p+C) \tau}{\gamma}<0
$$

makes $\dot{E}_{p}$ non-negative in a neighborhood of the equilibrium of interest. LaSalle's invariance principle can then be used to establish asymptotic stability of the equilibrium $\phi=0$ of (14) and the size of the basin of attraction. Summarizing, we have the following result.

Theorem 4. The equilibrium $\phi=0$ of the reduced shape equation (14) is asymptotically stable if $U_{p}^{\prime \prime}(0)$ is negative and conditions (16) and (17) hold.

\section{Stabilization of the Pendulum on a Rotor Arm}

Consider a planar pendulum attached to a horizontal rotor arm as shown in Figure 1. This mechanical system is studied in Åström and Furuta [1] and Bloch, Leonard, and Marsden [8]. The latter paper shows that matching conditions (4) cannot be satisfied for the pendulum on a rotor arm. As shown in [8], a more general controlled Lagrangian than (3) can be constructed for stabilization of the pendulum on a rotor arm. We show here that the relaxed matching is applicable as well and results in larger basin of attraction for the upward relative equilibrium of the pendulum.

The plane of the pendulum is orthogonal to the arm. The rotor arm is subject to a control torque $u$ that we intend to use for stabilizing the upward vertical relative equilibrium of the pendulum. As shown in the figure, the length of the pendulum is $l$, the pendulum bob mass is $m$, the length of the rotor arm is $R$, the mass

\footnotetext{
${ }^{2}$ This is true for the pendulum on a rotor arm and, more generally, for systems with $\gamma^{\prime}(\phi)$ vanishing at the relative equilibria.
} 
attached to the rotor arm is $M$, and the tilt of the pendulum measured from the upward vertical is $\phi$. The orientation of the rotor arm is given by the angle $s$. The

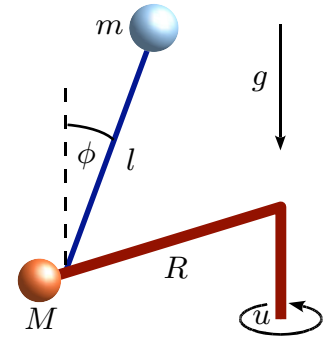

Figure 1. The pendulum on a rotor arm.

configuration space for this system is the two-dimensional torus parametrized by the angles $\phi$ and $s$.

The Lagrangian for this system is given by formula (11) with the kinetic energy metric coefficients $\alpha, \beta(\phi)$, and $\gamma(\phi)$ and the potential energy defined by

$\alpha=m l^{2}, \quad \beta(\phi)=m l R \cos \phi, \quad \gamma(\phi)=m l^{2} \sin ^{2} \phi+(m+M) R^{2}, \quad U(\phi)=m g l \cos \phi$.

This system is invariant with respect to rotations about the axis of the rotor arm, i.e., $s$ is a cyclic variable. The relative equilibria of the unforced $(u=0)$ system are

$$
\phi=\phi_{e}, \quad \dot{\phi}=0, \quad s=\omega t+s_{0}, \quad \dot{s}=\omega,
$$

where $\omega=$ const and where $\phi_{e}$ are roots of the equation

$$
\sin \phi\left(\omega^{2} \cos \phi+\frac{g}{l}\right)=0,
$$

see [8] for details. Equation (18) has two solutions $\phi_{e}=0, \pi$ if $\omega^{2}<g / l$. When $\omega^{2}>$ $g / l$, two additional solutions $\phi_{e}= \pm \arccos \left(-g /\left(\omega^{2} l\right)\right)$ appear; the corresponding relative equilibria are stable. The upright vertical relative equilibrium $\phi_{e}=0$ is always unstable. The relative equilibrium $\phi_{e}=\pi$ is stable if $\omega^{2}<g / l$. It becomes unstable when $\omega^{2}>g / l$.

We now stabilize the upward vertical relative equilibrium

$$
\phi_{e}=0, \quad \dot{s}=\omega
$$

of the pendulum on a rotor arm using techniques developed in Section 3. Note that condition (16) fails if $\phi= \pm \pi / 2$, and thus the range of $\phi$, for any choice of $\tau(\phi)$, cannot exceed the interval $(-\pi / 2, \pi / 2)$.

Below we assume that $c(\phi)$ is a negative constant, $c$. The amended potential and its derivative for the pendulum on the rotor arm are

$$
\begin{aligned}
& U_{p}=m g l \cos \phi-m g l-\int_{0}^{\phi} \frac{m l^{2} \sin x \cos x(p+c x)^{2}}{\left(m l^{2} \sin ^{2} x+(m+M) R^{2}\right)^{2}} d x, \\
& U_{p}^{\prime}=-\sin \phi\left[m g l+\frac{m l^{2} \cos \phi(p+c \phi)^{2}}{\left(m l^{2} \sin ^{2} \phi+(m+M) R^{2}\right)^{2}}\right] .
\end{aligned}
$$


Formulae (19) and (20) imply that the amended potential $U_{p}(\phi)$ is negative-definite throughout the interval $(-\pi / 2, \pi / 2)$ and has a single maximum at $\phi=0$.

Bloch, Leonard, and Marsden [8] define $\tau(\phi)$ by the formula

$$
\tau(\phi)=\kappa \frac{\beta(\phi)}{\gamma(\phi)}
$$

The maximal possible stabilization region for resulting controller is

$$
|\phi|<\arcsin \sqrt{\frac{R^{2}}{R^{2}+l^{2}}},
$$

(see [8] for details).

In this paper we define $\tau(\phi)$ by the formula

$$
\tau(\phi)=\varkappa \frac{m l^{2}}{\beta(\phi)},
$$

where $\kappa=$ const. This choice of $\tau(\phi)$ is not possible if the original matching techniques of [8] are used.

The first stability condition (16) becomes

$$
m l^{2}-m l^{2} \varkappa-\frac{m^{2} l^{2} R^{2} \cos ^{2} \phi}{(m+M) R^{2}+m l^{2} \sin ^{2} \phi}<0 .
$$

This stability condition is satisfied for the entire interval $|\phi|<\pi / 2$ if $\varkappa>1$. The second stability condition (17) is satisfied if $|\phi|<\pi / 2$ and $|p|$ is not too large. The torque produced by this controller is

$$
u=-\frac{d}{d t}\left[\frac{\varkappa \gamma(\phi) m l^{2} \dot{\phi}}{\beta(\phi)}\right]+c \dot{\phi}=-\frac{d}{d t}\left[\frac{\varkappa\left(m l^{2} \sin ^{2} \phi+(M+m) R^{2}\right) \dot{\phi}}{R \cos \phi}\right]+c \dot{\phi}
$$

Since the denominator in (22) vanishes as $\phi \rightarrow \pm \pi / 2$, formula (22) suggests that this controller may be capable of stabilizing the upward relative equilibrium from near horizontal initial tilt of the pendulum even if $\varkappa$ is not too large. This is confirmed by numerical simulation for the pendulum on a rotor arm with $m=1 \mathrm{~kg}$, $M=2 \mathrm{~kg}, l=1 \mathrm{~m}$, and $R=2$ mas discussed below. The gain parameters for simulation results in Figures 2 and 3 are $c=-50 \mathrm{~N} \cdot \mathrm{m} \cdot \mathrm{s}$ and $\varkappa=8 / 5$.

Figure 2 demonstrates stabilization of the pendulum by control torque $(22)$ from the state $\phi(0)=\pi / 4 \mathrm{rad}, \dot{\phi}(0)=0 \mathrm{rad} / \mathrm{s}, s(0)=0 \mathrm{rad}, \dot{s}(0)=0 \mathrm{rad} / \mathrm{s}$.

Figure 3 demonstrates stabilization of the pendulum by control torque $(22)$ from a nearly horizontal position $\phi(0)=\pi / 2-0.02 \mathrm{rad}$. The remaining initial conditions are $\dot{\phi}(0)=0 \mathrm{rad} / \mathrm{s}, s(0)=0 \mathrm{rad}, \dot{s}(0)=0 \mathrm{rad} / \mathrm{s}$.

Note that the controller in [8] cannot stabilize the pendulum from the initial tilt $\phi(0)=\pi / 2-0.02 \mathrm{rad}$ as this tilt fails to satisfy condition (21). The latter reads $|\phi|<\arcsin (2 / \sqrt{5}) \approx 1.10715$ for the system parameters used in the simulations. 

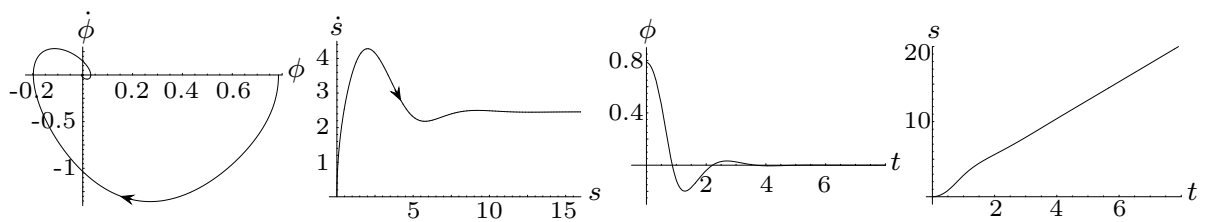

Figure 2. Asymptotic stabilization of the pendulum from $\phi(0)=\pi / 4$.

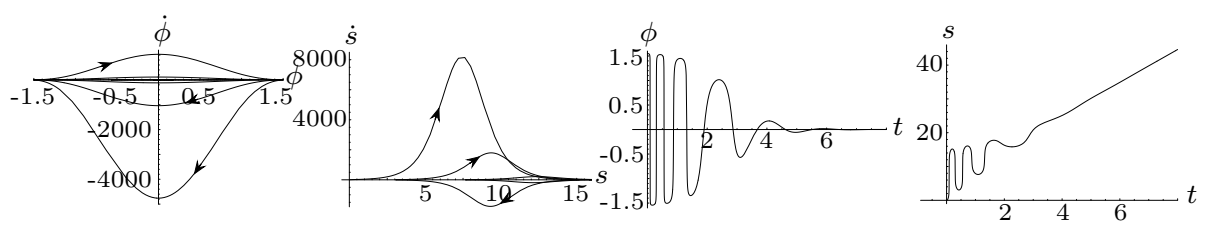

Figure 3. Asymptotic stabilization of the pendulum from $\phi(0)=\pi / 2-0.02$.

\section{Conclusions}

This paper developed some new relaxed matching techniques for mechanical systems with symmetry and has shown that these lead to an effective stabilizing controller design. The suggested formalism makes use of the intrinsic structure of mechanical systems and is less restrictive than the original matching procedure. Systems with non-commutative symmetry and combined kinetic and potential shaping for full state space stabilization will be treated in a forthcoming publication.

\section{Acknowledgments}

DAL and DVZ were partially supported by NSF grants DMS-0306017 and DMS0604108. AMB was supported in parts by NSF grants CMS-0408542 and DMS604307. JEM was partially supported by AFOSR grant FA9550-08-1-0173. 


\section{Bibliography}

[1] Åström, K. J., \& K. Furuta, Swinging up a pendulum by Energy Control, IFAC 13, 1996.

[2] Auckly, D., L. Kapitanski, \& W. White, Control of Nonlinear Underactuated Systems, Commun. Pure Appl. Math. 53, 2000, 354-369.

[3] Bloch, A.M., Nonholonomic Mechanics and Control, Interdisciplinary Appl. Math. 24, Springer-Verlag, 2003.

[4] Bloch, A. M., M. Leok, J. E. Marsden, \& D. V. Zenkov [2005], Controlled Lagrangians and Stabilization of the Discrete Cart-Pendulum System, Proc. CDC 44, 6579-6584.

[5] Bloch, A. M., M. Leok, J.E. Marsden, \& D. V. Zenkov [2006], Controlled Lagrangians and Potential Shaping for Stabilization of Discrete Mechanical Systems, Proc. CDC 45, 3333-3338.

[6] Bloch, A. M., N. Leonard, \& J.E. Marsden, Stabilization of Mechanical Systems Using Controlled Lagrangians, Proc. CDC 36, 1997, 2356-2361.

[7] Bloch, A. M., N. Leonard, \& J. E. Marsden, Matching and Stabilization by the Method of Controlled Lagrangians, Proc. CDC 37, 1998, 1446-1451.

[8] Bloch, A. M., N. Leonard, \& J.E. Marsden, Stabilization of the Pendulum on a Rotor Arm by the Method of Controlled Lagrangians, Proc. IEEE Int. Conf. Robotics Automat., 1999, 500-505.

[9] Bloch, A. M., N. Leonard, \& J. E. Marsden, Potential Shaping and the Method of Controlled Lagrangians, Proc. CDC 38, 1999, 1652-1657.

[10] Bloch, A. M., N. E. Leonard, \& J. E. Marsden, Controlled Lagrangians and the Stabilization of Mechanical Systems I: The First Matching Theorem, IEEE Trans. on Systems and Control 45, 2000, 2253-2270.

[11] Bloch, A. M., D-E. Chang, N.E. Leonard, \& J.E. Marsden, Controlled Lagrangians and the Stabilization of Mechanical Systems II: Potential Shaping, Trans. IEEE on Autom. Contr. 46, 2001, 1556-1571. 
[12] Chang, D-E., A. M. Bloch, N. E. Leonard, J. E. Marsden, \& C. Woolsey, The Equivalence of Controlled Lagrangian and Controlled Hamiltonian Systems, Control and the Calculus of Variations (special issue dedicated to J. L. Lions) 8, 2002, 393-422.

[13] Fujimoto, K., \& T. Sugie, Canonical Transformation and Stabilization of Generalized Hamiltonian Systems, Systems and Control Letters 42, 2001, 217-227.

[14] Hamberg, J., General Matching Conditions in the Theory of Controlled Lagrangians, Proc. CDC 38, 1999, 2519-2523.

[15] Hamberg, J., Controlled Lagrangians, Symmetries and Conditions for Strong Matching, In: Lagrangian and Hamiltonian Methods for Nonlinear Control, Elsevier, 2000.

[16] Long, D. A. \& D. V. Zenkov, Relaxed Matching for Stabilization of Relative Equilibria of Mechnical Systems, Proc. CDC 46, 2007, 6238-6243.

[17] Marsden, J. E., Lectures on Mechanics, London Mathematical Society Lecture Note Series 174, Cambridge University Press, 1992.

[18] Marsden, J. E. \& T.S. Ratiu, An Introduction to Mechanics and Symmetry. Texts in Appl. Math. 17, Springer-Verlag, 1999.

[19] Maschke, B., R. Ortega, \& A. van der Schaft, Energy-Based Lyapunov Functions for Forced Hamiltonian Systems with Dissipation, IEEE Trans. Automat. Control 45, 2001, 1498-1502.

[20] Ortega, R., M. W. Spong, F. Gómez-Estern, \& G. Blankenstein, Stabilization of a Class of Underactuated Mechanical Systems via Interconnection and Damping Assignment, IEEE Trans. Aut. Control 47, 2002, 1218-1233.

[21] Oziraner, A.S., On Asymptotic Stability and Instability Relative to a Part of Variables, J. Applied Math. Mech. 37, 1973, 623-629.

[22] Zenkov, D. V., A. M. Bloch, N.E. Leonard, \& J.E. Marsden, Matching and Stabilization of Low-Dimensional Nonholonomic Systems, Proc. CDC 39, 2000, 1289-1295.

[23] Zenkov, D. V., A. M. Bloch, \& J.E. Marsden, Flat Nonholonomic Matching, Proc. ACC, 2002, 2812-2817. 